Supporting information for

\title{
[Application of Low-Field Nuclear Magnetic Resonance (LFNMR) in Characterizing the Dissociation of Gas Hydrate in the Porous Media]
}

\author{
Yongchao Zhang ${ }^{1,2}$, Lele Liu ${ }^{1,2}$, Daigang Wang ${ }^{3}$, Pengfei Chen ${ }^{4}$, Zhun Zhang ${ }^{4}$, Qingguo Meng ${ }^{1,2}$, and Changling \\ $\operatorname{Liu}^{1,2, *}$ \\ ${ }^{1}$ The Key Laboratory of Gas Hydrate, Ministry of Natural Resources, Qingdao Institute of Marine Geology, Qingdao \\ 266071, PR China \\ ${ }^{2}$ Laboratory for Marine Mineral Resources, Pilot National Laboratory for Marine Science and Technology, Qingdao \\ 266071, PR China \\ ${ }^{3}$ Institute of Unconventional Oil and Gas Science and Technology Research, China University of Petroleum, Beijing \\ 100249, PR China \\ ${ }^{4}$ Faculty of Engineering, China University of Geosciences, Wuhan 430074, China
}

*Corresponding author: qdliuchangling@163.com (C. Liu)

\section{Contents of this file:}

Table S1, Table S2, Table S3, Table S4, and Table S5

\section{Introduction:}

The main contents in this supporting information include:

Table S1: Data of T2 spectra under the condition of fully saturated for the three sand samples Table S2: Data of T2 spectra obtained from different hydrate dissociation time for Sample 1 Table S3: Data of T2 spectra obtained from different hydrate dissociation time for Sample 2 Table S4: Data of T2 spectra obtained from different hydrate dissociation time for Sample 3 Table S5: Calculation results of the fractal dimension for the three samples 
Table S1: Data of T2 spectra under the condition of fully saturated for the three sand samples

\begin{tabular}{|c|c|c|c|c|c|c|c|}
\hline Time (ms) & Sample 1 & SAMPLE 2 & SAMPLE 3 & Time (ms) & Sample 1 & Sample 2 & Sample 3 \\
\hline 0.01 & 0.00 & 0.00 & 0.00 & 10.72 & 511.41 & 250.19 & 218.76 \\
\hline 0.01 & 0.00 & 0.00 & 0.00 & 12.33 & 578.84 & 289.65 & 234.12 \\
\hline 0.01 & 0.00 & 0.00 & 0.00 & 14.17 & 653.53 & 337.00 & 249.47 \\
\hline 0.02 & 0.00 & 0.00 & 0.00 & 16.30 & 735.48 & 389.88 & 275.05 \\
\hline 0.02 & 0.00 & 0.00 & 0.00 & 18.74 & 821.82 & 451.44 & 312.15 \\
\hline 0.02 & 0.00 & 0.00 & 0.00 & 21.54 & 917.50 & 521.69 & 360.77 \\
\hline 0.02 & 0.00 & 0.00 & 0.00 & 24.77 & 1011.13 & 602.98 & 424.73 \\
\hline 0.03 & 0.00 & 0.00 & 0.00 & 28.48 & 1103.72 & 687.43 & 510.45 \\
\hline 0.03 & 0.00 & 0.00 & 0.00 & 32.75 & 1191.14 & 776.61 & 607.68 \\
\hline 0.04 & 0.00 & 0.00 & 0.00 & 37.65 & 1282.48 & 866.58 & 717.70 \\
\hline 0.04 & 0.00 & 0.00 & 0.00 & 43.29 & 1373.82 & 954.19 & 837.95 \\
\hline 0.05 & 0.00 & 0.00 & 0.00 & 49.77 & 1473.45 & 1038.64 & 965.88 \\
\hline 0.05 & 0.00 & 0.00 & 0.00 & 57.22 & 1579.31 & 1123.88 & 1100.21 \\
\hline 0.06 & 0.00 & 0.00 & 0.00 & 65.79 & 1688.29 & 1209.11 & 1243.50 \\
\hline 0.07 & 0.00 & 0.00 & 0.00 & 75.65 & 1797.26 & 1291.20 & 1395.73 \\
\hline 0.08 & 0.00 & 0.00 & 0.00 & 86.97 & 1904.16 & 1372.49 & 1553.09 \\
\hline 0.09 & 0.00 & 0.00 & 0.00 & 100.00 & 2002.76 & 1441.15 & 1715.56 \\
\hline 0.11 & 0.00 & 0.00 & 0.00 & 114.98 & 2088.91 & 1499.55 & 1876.76 \\
\hline 0.12 & 0.00 & 0.00 & 0.51 & 132.19 & 2165.73 & 1545.33 & 2034.11 \\
\hline 0.14 & 0.00 & 0.00 & 1.98 & 151.99 & 2229.06 & 1578.47 & 2181.24 \\
\hline 0.16 & 0.00 & 0.00 & 5.09 & 174.75 & 2276.84 & 1595.05 & 2311.73 \\
\hline 0.19 & 0.00 & 0.00 & 9.21 & 200.92 & 2308.01 & 1598.21 & 2420.47 \\
\hline 0.22 & 0.00 & 0.00 & 14.53 & 231.01 & 2323.62 & 1582.42 & 2499.79 \\
\hline 0.25 & 0.00 & 0.00 & 20.41 & 265.61 & 2323.65 & 1540.59 & 2548.56 \\
\hline 0.28 & 0.00 & 0.18 & 26.62 & 305.39 & 2302.35 & 1471.14 & 2565.92 \\
\hline 0.33 & 0.00 & 0.58 & 32.46 & 351.12 & 2256.55 & 1374.06 & 2542.89 \\
\hline
\end{tabular}




\begin{tabular}{|c|c|c|c|c|c|c|c|}
\hline 0.38 & 0.00 & 1.34 & 39.86 & 403.70 & 2199.16 & 1259.62 & 2473.81 \\
\hline 0.43 & 0.00 & 2.71 & 47.33 & 464.16 & 2111.15 & 1128.61 & 2361.23 \\
\hline 0.50 & 0.00 & 4.93 & 55.01 & 533.67 & 1991.55 & 980.23 & 2207.54 \\
\hline 0.57 & 0.00 & 8.19 & 61.41 & 613.59 & 1862.04 & 813.70 & 2003.41 \\
\hline 0.66 & 0.00 & 12.89 & 70.36 & 705.48 & 1659.73 & 643.23 & 1764.18 \\
\hline 0.76 & 0.88 & 19.28 & 76.76 & 811.13 & 1462.66 & 466.44 & 1491.68 \\
\hline 0.87 & 3.72 & 27.48 & 85.71 & 932.60 & 1250.22 & 295.96 & 1200.00 \\
\hline 1.00 & 9.53 & 37.44 & 94.67 & 1072.27 & 1006.23 & 164.16 & 885.29 \\
\hline 1.15 & 18.82 & 48.80 & 106.18 & 1232.85 & 772.82 & 76.56 & 569.30 \\
\hline 1.32 & 32.11 & 60.95 & 116.42 & 1417.47 & 508.29 & 22.89 & 236.67 \\
\hline 1.52 & 49.21 & 73.11 & 129.21 & 1629.75 & 280.08 & 0.00 & 0.00 \\
\hline 1.75 & 70.10 & 84.50 & 137.29 & 1873.82 & 88.17 & 0.00 & 0.00 \\
\hline 2.01 & 93.68 & 94.55 & 144.88 & 2154.43 & 15.56 & 0.00 & 0.00 \\
\hline 2.31 & 118.29 & 102.64 & 150.32 & 2477.08 & 0.00 & 0.00 & 0.00 \\
\hline 2.66 & 144.42 & 108.28 & 151.60 & 2848.04 & 0.00 & 0.00 & 0.00 \\
\hline 3.05 & 172.09 & 112.65 & 152.88 & 3274.55 & 0.00 & 0.00 & 0.00 \\
\hline 3.51 & 198.14 & 116.16 & 155.04 & 3764.94 & 0.00 & 0.00 & 0.00 \\
\hline 4.04 & 223.74 & 119.96 & 158.96 & 4328.76 & 0.00 & 0.00 & 0.00 \\
\hline 4.64 & 252.07 & 126.28 & 163.75 & 4977.02 & 0.00 & 0.00 & 0.00 \\
\hline 5.34 & 282.16 & 135.75 & 170.15 & 5722.37 & 0.00 & 0.00 & 0.00 \\
\hline 6.14 & 314.32 & 149.17 & 176.55 & 6579.33 & 0.00 & 0.00 & 0.00 \\
\hline 7.05 & 352.70 & 166.53 & 186.78 & 7564.63 & 0.00 & 0.00 & 0.00 \\
\hline 8.11 & 400.41 & 189.42 & 195.74 & 8697.49 & 0.00 & 0.00 & 0.00 \\
\hline 9.33 & 453.32 & 217.04 & 204.69 & 10000.00 & 0.00 & 0.00 & 0.00 \\
\hline
\end{tabular}


Table S2: Data of T2 spectra obtained from different hydrate dissociation time for Sample 1

\begin{tabular}{|c|c|c|c|c|c|c|c|c|c|c|}
\hline Time $(\mathrm{ms})$ & D1 & D2 & D3 & D4 & D5 & D6 & D7 & D8 & D9 & D10 \\
\hline 0.01 & 0.00 & 0.00 & 0.00 & 0.00 & 0.00 & 0.00 & 0.00 & 0.00 & 0.00 & 0.00 \\
\hline 0.01 & 0.00 & 0.00 & 0.00 & 0.00 & 0.00 & 0.00 & 0.00 & 0.00 & 0.00 & 0.00 \\
\hline 0.01 & 0.00 & 0.00 & 0.00 & 0.00 & 0.00 & 0.00 & 0.00 & 0.00 & 0.00 & 0.00 \\
\hline 0.02 & 0.00 & 0.00 & 0.00 & 0.00 & 0.00 & 0.00 & 0.00 & 0.00 & 0.00 & 0.00 \\
\hline 0.02 & 0.00 & 0.00 & 0.00 & 0.00 & 0.00 & 0.00 & 0.00 & 0.00 & 0.00 & 0.00 \\
\hline 0.02 & 0.00 & 0.00 & 0.00 & 0.00 & 0.00 & 0.00 & 0.00 & 0.00 & 0.00 & 0.00 \\
\hline 0.02 & 0.00 & 0.00 & 0.00 & 0.00 & 0.00 & 0.00 & 0.00 & 0.00 & 0.00 & 0.00 \\
\hline 0.03 & 0.00 & 0.00 & 0.00 & 0.00 & 0.00 & 0.00 & 0.00 & 0.00 & 0.00 & 0.00 \\
\hline 0.03 & 0.00 & 0.00 & 0.00 & 0.00 & 0.00 & 0.00 & 0.00 & 0.00 & 0.00 & 0.00 \\
\hline 0.04 & 0.00 & 0.00 & 0.00 & 0.00 & 0.00 & 0.00 & 0.00 & 0.00 & 0.00 & 0.00 \\
\hline 0.04 & 0.00 & 0.00 & 0.00 & 0.00 & 0.00 & 0.00 & 0.00 & 0.00 & 0.00 & 0.00 \\
\hline 0.05 & 0.00 & 0.00 & 0.00 & 0.00 & 0.00 & 0.00 & 0.00 & 0.00 & 0.00 & 0.00 \\
\hline 0.05 & 0.00 & 0.00 & 0.00 & 0.00 & 0.00 & 0.00 & 0.00 & 0.00 & 0.00 & 0.00 \\
\hline 0.06 & 0.00 & 0.00 & 0.00 & 0.00 & 0.00 & 0.00 & 0.00 & 0.00 & 0.00 & 0.00 \\
\hline 0.07 & 0.00 & 0.00 & 0.00 & 0.00 & 0.00 & 0.00 & 0.00 & 0.00 & 0.00 & 0.00 \\
\hline 0.08 & 0.00 & 0.00 & 0.00 & 0.00 & 0.00 & 0.00 & 0.00 & 0.00 & 0.00 & 0.00 \\
\hline 0.09 & 0.00 & 0.00 & 0.00 & 0.00 & 0.00 & 0.00 & 0.00 & 0.00 & 0.00 & 0.00 \\
\hline 0.11 & 0.00 & 0.00 & 0.00 & 0.00 & 0.00 & 0.00 & 0.00 & 0.00 & 0.00 & 0.00 \\
\hline 0.12 & 0.00 & 0.00 & 0.00 & 0.00 & 0.00 & 0.00 & 0.00 & 0.00 & 0.00 & 0.00 \\
\hline 0.14 & 0.00 & 0.00 & 0.00 & 0.00 & 0.00 & 0.00 & 0.00 & 0.00 & 0.00 & 0.00 \\
\hline 0.16 & 0.00 & 0.00 & 0.00 & 0.00 & 0.00 & 0.00 & 0.00 & 0.00 & 0.00 & 0.00 \\
\hline 0.19 & 0.00 & 0.00 & 0.00 & 0.00 & 0.00 & 0.00 & 0.00 & 0.00 & 0.00 & 0.00 \\
\hline 0.22 & 0.00 & 0.00 & 0.00 & 0.00 & 0.00 & 0.00 & 0.00 & 0.00 & 0.00 & 0.00 \\
\hline 0.25 & 0.00 & 0.00 & 0.00 & 0.00 & 0.00 & 0.00 & 0.00 & 0.00 & 0.00 & 0.00 \\
\hline 0.28 & 0.00 & 0.00 & 0.00 & 0.00 & 0.00 & 0.00 & 0.00 & 0.00 & 0.00 & 0.00 \\
\hline 0.33 & 0.00 & 0.00 & 0.00 & 0.00 & 0.00 & 0.00 & 0.00 & 0.00 & 0.00 & 0.00 \\
\hline
\end{tabular}




\begin{tabular}{|c|c|c|c|c|c|c|c|c|c|c|}
\hline 0.38 & 0.00 & 0.00 & 0.00 & 0.00 & 0.00 & 0.00 & 0.00 & 0.00 & 0.00 & 0.00 \\
\hline 0.43 & 0.00 & 0.00 & 0.00 & 0.00 & 0.00 & 0.00 & 0.00 & 0.00 & 0.00 & 0.00 \\
\hline 0.50 & 0.00 & 0.00 & 0.00 & 0.00 & 0.00 & 0.00 & 0.00 & 0.00 & 0.00 & 0.00 \\
\hline 0.57 & 0.00 & 0.00 & 0.00 & 0.00 & 0.00 & 0.00 & 0.00 & 0.00 & 0.00 & 0.00 \\
\hline 0.66 & 0.00 & 0.00 & 0.00 & 0.00 & 0.00 & 0.00 & 0.00 & 0.00 & 0.00 & 0.00 \\
\hline 0.76 & 0.00 & 0.00 & 0.00 & 0.00 & 0.00 & 0.00 & 0.00 & 0.00 & 0.00 & 0.00 \\
\hline 0.87 & 0.00 & 0.00 & 0.00 & 0.00 & 0.00 & 0.00 & 0.00 & 0.00 & 0.00 & 0.00 \\
\hline 1.00 & 0.00 & 0.00 & 0.00 & 0.00 & 0.00 & 0.00 & 0.00 & 0.00 & 0.00 & 1.19 \\
\hline 1.15 & 0.00 & 0.00 & 0.00 & 0.00 & 0.00 & 0.00 & 1.05 & 1.85 & 2.64 & 10.56 \\
\hline 1.32 & 0.00 & 0.00 & 1.85 & 2.64 & 3.43 & 3.96 & 9.23 & 11.52 & 13.80 & 26.57 \\
\hline 1.52 & 5.71 & 7.74 & 11.52 & 13.80 & 16.09 & 19.43 & 24.88 & 28.22 & 31.30 & 42.02 \\
\hline 1.75 & 19.43 & 23.82 & 28.22 & 31.56 & 34.90 & 39.74 & 42.29 & 45.10 & 48.97 & 57.76 \\
\hline 2.01 & 37.98 & 42.55 & 45.10 & 48.18 & 51.25 & 55.12 & 58.20 & 62.33 & 67.43 & 74.29 \\
\hline 2.31 & 54.59 & 58.73 & 62.86 & 65.67 & 68.75 & 72.62 & 75.43 & 79.74 & 87.21 & 95.21 \\
\hline 2.66 & 71.30 & 73.85 & 78.42 & 82.55 & 85.63 & 90.20 & 94.33 & 99.52 & 107.16 & 114.94 \\
\hline 3.05 & 85.36 & 89.23 & 93.10 & 97.67 & 100.75 & 104.88 & 109.45 & 113.76 & 130.12 & 137.73 \\
\hline 3.51 & 98.99 & 104.35 & 107.96 & 112.09 & 114.90 & 117.71 & 121.58 & 137.67 & 152.92 & 160.54 \\
\hline 4.04 & 114.64 & 121.05 & 126.42 & 128.70 & 132.31 & 134.59 & 137.67 & 156.67 & 175.72 & 187.16 \\
\hline 4.64 & 130.73 & 136.62 & 142.77 & 146.90 & 152.79 & 156.66 & 162.81 & 183.28 & 202.33 & 213.76 \\
\hline 5.34 & 138.33 & 144.48 & 150.90 & 157.05 & 163.96 & 172.31 & 181.78 & 206.08 & 228.93 & 240.36 \\
\hline 6.14 & 145.93 & 152.35 & 159.03 & 167.21 & 175.12 & 187.96 & 200.75 & 228.88 & 251.73 & 266.98 \\
\hline 7.05 & 152.84 & 160.92 & 168.62 & 180.40 & 192.22 & 214.15 & 230.37 & 251.68 & 288.12 & 302.39 \\
\hline 8.11 & 159.74 & 169.49 & 178.20 & 193.58 & 209.32 & 240.35 & 259.99 & 282.09 & 302.56 & 348.24 \\
\hline 9.33 & 166.02 & 177.93 & 188.31 & 211.03 & 232.57 & 273.58 & 298.91 & 327.33 & 351.63 & 405.23 \\
\hline 10.72 & 172.31 & 186.37 & 198.42 & 228.48 & 255.82 & 306.81 & 337.82 & 373.42 & 404.12 & 468.48 \\
\hline 12.33 & 175.12 & 192.18 & 208.22 & 247.96 & 282.42 & 341.89 & 381.10 & 427.60 & 462.47 & 535.87 \\
\hline 14.17 & 177.93 & 197.98 & 218.02 & 267.43 & 309.01 & 376.97 & 424.39 & 481.78 & 520.82 & 603.25 \\
\hline 16.30 & 177.06 & 200.26 & 226.02 & 285.49 & 334.11 & 410.29 & 467.57 & 536.32 & 582.61 & 677.45 \\
\hline
\end{tabular}




\begin{tabular}{|c|c|c|c|c|c|c|c|c|c|c|}
\hline 18.74 & 176.18 & 202.55 & 234.02 & 303.56 & 359.21 & 443.60 & 510.75 & 590.85 & 644.40 & 751.65 \\
\hline 21.54 & 170.51 & 201.27 & 241.54 & 318.86 & 383.21 & 474.86 & 552.06 & 644.13 & 708.84 & 832.62 \\
\hline 24.77 & 164.84 & 200.00 & 249.06 & 334.15 & 407.21 & 506.11 & 593.37 & 697.40 & 773.27 & 913.58 \\
\hline 28.48 & 155.12 & 193.32 & 252.97 & 344.88 & 425.36 & 532.31 & 630.02 & 746.39 & 836.41 & 996.13 \\
\hline 32.75 & 145.41 & 186.64 & 256.88 & 355.60 & 443.52 & 558.51 & 666.67 & 795.39 & 899.56 & 1078.68 \\
\hline 37.65 & 132.04 & 177.19 & 255.60 & 358.81 & 457.85 & 576.79 & 695.78 & 836.43 & 954.21 & 1151.52 \\
\hline 43.29 & 118.68 & 167.74 & 254.33 & 362.02 & 472.18 & 595.08 & 724.89 & 877.46 & 1008.87 & 1224.35 \\
\hline 49.77 & 106.95 & 157.45 & 246.42 & 357.19 & 478.59 & 606.42 & 743.61 & 906.85 & 1051.86 & 1284.53 \\
\hline 57.22 & 95.21 & 147.16 & 238.51 & 352.35 & 485.01 & 617.76 & 762.33 & 936.24 & 1094.85 & 1344.70 \\
\hline 65.79 & 84.75 & 136.53 & 226.68 & 342.20 & 482.29 & 619.25 & 768.10 & 950.50 & 1120.16 & 1383.17 \\
\hline 75.65 & 74.29 & 125.89 & 214.86 & 332.04 & 479.56 & 620.75 & 773.87 & 964.77 & 1145.47 & 1421.63 \\
\hline 86.97 & 65.89 & 113.85 & 199.08 & 316.79 & 460.88 & 607.65 & 764.56 & 960.86 & 1149.37 & 1433.67 \\
\hline 100.00 & 57.49 & 101.80 & 183.30 & 301.54 & 442.20 & 594.55 & 755.25 & 956.95 & 1153.28 & 1445.71 \\
\hline 114.98 & 47.82 & 89.23 & 165.14 & 277.67 & 413.85 & 564.22 & 729.36 & 932.48 & 1136.45 & 1435.12 \\
\hline 132.19 & 38.15 & 76.66 & 146.99 & 253.80 & 385.49 & 533.89 & 703.48 & 908.01 & 1119.62 & 1424.53 \\
\hline 151.99 & 28.04 & 64.88 & 127.96 & 221.89 & 347.82 & 490.02 & 660.24 & 861.42 & 1077.19 & 1382.73 \\
\hline 174.75 & 17.93 & 53.10 & 108.92 & 189.98 & 310.15 & 446.15 & 617.00 & 814.83 & 1034.76 & 1340.92 \\
\hline 200.92 & 10.42 & 40.75 & 89.98 & 158.51 & 271.16 & 390.86 & 556.06 & 744.74 & 964.22 & 1264.04 \\
\hline 231.01 & 2.90 & 28.40 & 71.03 & 127.03 & 232.18 & 335.56 & 495.13 & 674.66 & 893.67 & 1187.17 \\
\hline 265.61 & 1.45 & 18.73 & 52.18 & 100.26 & 187.52 & 271.74 & 415.95 & 582.67 & 796.44 & 1076.57 \\
\hline 305.39 & 0.00 & 9.05 & 33.32 & 73.49 & 142.86 & 207.91 & 336.77 & 490.68 & 699.20 & 965.98 \\
\hline 351.12 & 0.00 & 4.53 & 20.92 & 52.66 & 104.88 & 154.07 & 272.47 & 410.87 & 589.69 & 840.97 \\
\hline 403.70 & 0.00 & 0.00 & 8.53 & 31.82 & 66.90 & 100.22 & 200.49 & 320.76 & 480.18 & 715.96 \\
\hline 464.16 & 0.00 & 0.00 & 4.26 & 18.64 & 41.49 & 65.93 & 139.54 & 235.15 & 374.17 & 584.90 \\
\hline 533.67 & 0.00 & 0.00 & 0.00 & 5.45 & 16.09 & 31.65 & 79.09 & 146.53 & 268.15 & 453.84 \\
\hline 613.59 & 0.00 & 0.00 & 0.00 & 2.73 & 8.70 & 18.20 & 42.47 & 86.75 & 191.03 & 340.46 \\
\hline 705.48 & 0.00 & 0.00 & 0.00 & 0.00 & 1.32 & 4.75 & 18.85 & 36.96 & 113.92 & 227.07 \\
\hline 811.13 & 0.00 & 0.00 & 0.00 & 0.00 & 0.00 & 2.37 & 7.67 & 16.98 & 53.95 & 148.94 \\
\hline
\end{tabular}




\begin{tabular}{|l|l|l|l|l|l|l|l|l|l|l|}
\hline 932.60 & 0.00 & 0.00 & 0.00 & 0.00 & 0.00 & 0.00 & 1.50 & 6.99 & 27.99 & 70.80 \\
\hline 1072.27 & 0.00 & 0.00 & 0.00 & 0.00 & 0.00 & 0.00 & 0.00 & 2.48 & 12.96 & 37.42 \\
\hline 1232.85 & 0.00 & 0.00 & 0.00 & 0.00 & 0.00 & 0.00 & 0.00 & 0.00 & 1.93 & 4.03 \\
\hline 1417.47 & 0.00 & 0.00 & 0.00 & 0.00 & 0.00 & 0.00 & 0.00 & 0.00 & 0.00 & 2.01 \\
\hline 1629.75 & 0.00 & 0.00 & 0.00 & 0.00 & 0.00 & 0.00 & 0.00 & 0.00 & 0.00 & 0.00 \\
\hline 1873.82 & 0.00 & 0.00 & 0.00 & 0.00 & 0.00 & 0.00 & 0.00 & 0.00 & 0.00 & 0.00 \\
\hline 2154.43 & 0.00 & 0.00 & 0.00 & 0.00 & 0.00 & 0.00 & 0.00 & 0.00 & 0.00 & 0.00 \\
\hline 2477.08 & 0.00 & 0.00 & 0.00 & 0.00 & 0.00 & 0.00 & 0.00 & 0.00 & 0.00 & 0.00 \\
\hline 2848.04 & 0.00 & 0.00 & 0.00 & 0.00 & 0.00 & 0.00 & 0.00 & 0.00 & 0.00 & 0.00 \\
\hline 3274.55 & 0.00 & 0.00 & 0.00 & 0.00 & 0.00 & 0.00 & 0.00 & 0.00 & 0.00 & 0.00 \\
\hline 3764.94 & 0.00 & 0.00 & 0.00 & 0.00 & 0.00 & 0.00 & 0.00 & 0.00 & 0.00 & 0.00 \\
\hline 4328.76 & 0.00 & 0.00 & 0.00 & 0.00 & 0.00 & 0.00 & 0.00 & 0.00 & 0.00 & 0.00 \\
\hline 4977.02 & 0.00 & 0.00 & 0.00 & 0.00 & 0.00 & 0.00 & 0.00 & 0.00 & 0.00 & 0.00 \\
\hline 5722.37 & 0.00 & 0.00 & 0.00 & 0.00 & 0.00 & 0.00 & 0.00 & 0.00 & 0.00 & 0.00 \\
\hline 6579.33 & 0.00 & 0.00 & 0.00 & 0.00 & 0.00 & 0.00 & 0.00 & 0.00 & 0.00 & 0.00 \\
\hline 7564.63 & 0.00 & 0.00 & 0.00 & 0.00 & 0.00 & 0.00 & 0.00 & 0.00 & 0.00 & 0.00 \\
\hline 8697.49 & 0.00 & 0.00 & 0.00 & 0.00 & 0.00 & 0.00 & 0.00 & 0.00 & 0.00 & 0.00 \\
\hline 10000.00 & 0.00 & 0.00 & 0.00 & 0.00 & 0.00 & 0.00 & 0.00 & 0.00 & 0.00 & 0.00 \\
\hline
\end{tabular}


Table S3: Data of T2 spectra obtained from different hydrate dissociation time for Sample 2

\begin{tabular}{|c|c|c|c|c|c|c|c|c|c|c|}
\hline Time(ms) & D1 & D2 & D3 & D4 & D5 & D6 & D7 & D8 & D9 & D10 \\
\hline 0.01 & 0.00 & 0.00 & 0.00 & 0.00 & 0.00 & 0.00 & 0.00 & 0.00 & 0.00 & 0.00 \\
\hline 0.01 & 0.00 & 0.00 & 0.00 & 0.00 & 0.00 & 0.00 & 0.00 & 0.00 & 0.00 & 0.00 \\
\hline 0.01 & 0.00 & 0.00 & 0.00 & 0.00 & 0.00 & 0.00 & 0.00 & 0.00 & 0.00 & 0.00 \\
\hline 0.02 & 0.00 & 0.00 & 0.00 & 0.00 & 0.00 & 0.00 & 0.00 & 0.00 & 0.00 & 0.00 \\
\hline 0.02 & 0.00 & 0.00 & 0.00 & 0.00 & 0.00 & 0.00 & 0.00 & 0.00 & 0.00 & 0.00 \\
\hline 0.02 & 0.00 & 0.00 & 0.00 & 0.00 & 0.00 & 0.00 & 0.00 & 0.00 & 0.00 & 0.00 \\
\hline 0.02 & 0.00 & 0.00 & 0.00 & 0.00 & 0.00 & 0.00 & 0.00 & 0.00 & 0.00 & 0.00 \\
\hline 0.03 & 0.00 & 0.00 & 0.00 & 0.00 & 0.00 & 0.00 & 0.00 & 0.00 & 0.00 & 0.00 \\
\hline 0.03 & 0.00 & 0.00 & 0.00 & 0.00 & 0.00 & 0.00 & 0.00 & 0.00 & 0.00 & 0.00 \\
\hline 0.04 & 0.00 & 0.00 & 0.00 & 0.00 & 0.00 & 0.00 & 0.00 & 0.00 & 0.00 & 0.00 \\
\hline 0.04 & 0.00 & 0.00 & 0.00 & 0.00 & 0.00 & 0.00 & 0.00 & 0.00 & 0.00 & 0.00 \\
\hline 0.05 & 0.00 & 0.00 & 0.00 & 0.00 & 0.00 & 0.00 & 0.00 & 0.00 & 0.00 & 0.00 \\
\hline 0.05 & 0.00 & 0.00 & 0.00 & 0.00 & 0.00 & 0.00 & 0.00 & 0.00 & 0.00 & 0.00 \\
\hline 0.06 & 0.00 & 0.00 & 0.00 & 0.00 & 0.00 & 0.00 & 0.00 & 0.00 & 0.00 & 0.00 \\
\hline 0.07 & 0.00 & 0.00 & 0.00 & 0.00 & 0.00 & 0.00 & 0.00 & 0.00 & 0.00 & 0.00 \\
\hline 0.08 & 0.00 & 0.00 & 0.00 & 0.00 & 0.00 & 0.00 & 0.00 & 0.00 & 0.00 & 0.00 \\
\hline 0.09 & 0.00 & 0.00 & 0.00 & 0.00 & 0.00 & 0.00 & 0.00 & 0.00 & 0.00 & 0.00 \\
\hline 0.11 & 0.00 & 0.00 & 0.00 & 0.00 & 0.00 & 0.00 & 0.00 & 0.00 & 0.00 & 0.00 \\
\hline 0.12 & 0.00 & 0.00 & 0.00 & 0.00 & 0.00 & 0.00 & 0.00 & 0.00 & 0.00 & 0.00 \\
\hline 0.14 & 0.00 & 0.00 & 0.00 & 0.00 & 0.00 & 0.00 & 0.00 & 0.00 & 0.00 & 0.00 \\
\hline 0.16 & 0.00 & 0.00 & 0.00 & 0.00 & 0.00 & 0.00 & 0.00 & 0.00 & 0.00 & 0.00 \\
\hline 0.19 & 0.00 & 0.00 & 0.00 & 0.00 & 0.00 & 0.00 & 0.00 & 0.00 & 0.00 & 0.00 \\
\hline 0.22 & 0.00 & 0.00 & 0.00 & 0.00 & 0.00 & 0.00 & 0.00 & 0.00 & 0.00 & 0.00 \\
\hline 0.25 & 0.00 & 0.00 & 0.00 & 0.00 & 0.00 & 0.00 & 0.00 & 0.00 & 0.00 & 0.00 \\
\hline 0.28 & 0.00 & 0.00 & 0.00 & 0.00 & 0.00 & 0.00 & 0.00 & 0.00 & 0.00 & 0.00 \\
\hline 0.33 & 0.00 & 0.00 & 0.00 & 0.00 & 0.00 & 0.00 & 0.00 & 0.00 & 0.00 & 0.00 \\
\hline
\end{tabular}




\begin{tabular}{|c|c|c|c|c|c|c|c|c|c|c|}
\hline 0.38 & 0.00 & 0.00 & 0.00 & 0.00 & 0.00 & 0.00 & 0.00 & 0.00 & 0.00 & 0.00 \\
\hline 0.43 & 0.00 & 0.00 & 0.00 & 0.00 & 0.00 & 0.00 & 0.00 & 0.00 & 0.00 & 0.00 \\
\hline 0.50 & 0.00 & 0.00 & 0.00 & 0.00 & 0.00 & 0.00 & 0.00 & 0.00 & 0.00 & 0.00 \\
\hline 0.57 & 0.00 & 0.00 & 0.00 & 0.00 & 0.00 & 0.00 & 0.00 & 0.00 & 0.00 & 0.16 \\
\hline 0.66 & 0.00 & 0.00 & 0.00 & 0.00 & 0.00 & 0.00 & 0.00 & 0.00 & 0.40 & 0.93 \\
\hline 0.76 & 0.00 & 0.00 & 0.00 & 0.00 & 0.00 & 0.40 & 0.81 & 1.21 & 2.02 & 3.07 \\
\hline 0.87 & 0.00 & 0.00 & 0.40 & 0.81 & 1.21 & 2.02 & 2.83 & 3.64 & 4.86 & 7.54 \\
\hline 1.00 & 2.02 & 2.83 & 3.64 & 4.86 & 6.07 & 7.69 & 8.91 & 10.53 & 12.15 & 15.19 \\
\hline 1.15 & 8.91 & 10.12 & 11.34 & 12.96 & 14.98 & 17.00 & 18.62 & 20.65 & 22.67 & 26.18 \\
\hline 1.32 & 19.43 & 21.05 & 22.67 & 24.70 & 27.13 & 29.55 & 31.58 & 34.01 & 36.03 & 39.97 \\
\hline 1.52 & 32.39 & 34.41 & 36.44 & 38.87 & 41.70 & 44.13 & 46.15 & 48.58 & 50.61 & 55.34 \\
\hline 1.75 & 47.77 & 50.20 & 52.23 & 54.66 & 57.49 & 59.92 & 61.94 & 64.37 & 66.40 & 70.46 \\
\hline 2.01 & 62.35 & 64.37 & 66.40 & 68.42 & 70.85 & 72.87 & 74.90 & 76.92 & 78.95 & 83.72 \\
\hline 2.31 & 72.47 & 74.49 & 76.52 & 78.54 & 80.57 & 82.59 & 84.62 & 86.64 & 88.66 & 94.24 \\
\hline 2.66 & 77.73 & 79.76 & 81.78 & 84.21 & 86.64 & 89.07 & 91.09 & 93.12 & 95.14 & 101.80 \\
\hline 3.05 & 78.95 & 81.38 & 83.81 & 86.64 & 89.47 & 92.71 & 95.14 & 97.17 & 99.60 & 106.60 \\
\hline 3.51 & 76.52 & 79.35 & 82.19 & 85.43 & 89.07 & 93.12 & 96.36 & 98.79 & 101.62 & 109.59 \\
\hline 4.04 & 72.47 & 75.71 & 78.95 & 82.59 & 87.04 & 91.90 & 95.95 & 98.79 & 102.43 & 111.78 \\
\hline 4.64 & 68.02 & 71.66 & 75.30 & 79.76 & 85.02 & 90.69 & 95.55 & 98.79 & 103.64 & 114.24 \\
\hline 5.34 & 65.59 & 70.04 & 75.30 & 80.16 & 85.83 & 92.31 & 97.98 & 102.02 & 108.10 & 118.03 \\
\hline 6.14 & 65.99 & 70.85 & 78.14 & 83.81 & 89.88 & 97.17 & 103.64 & 109.31 & 117.00 & 124.28 \\
\hline 7.05 & 69.23 & 74.49 & 83.81 & 91.09 & 97.57 & 106.88 & 114.17 & 121.05 & 130.77 & 133.84 \\
\hline 8.11 & 74.49 & 80.16 & 91.90 & 102.02 & 110.12 & 121.46 & 129.96 & 138.46 & 150.20 & 147.74 \\
\hline 9.33 & 80.97 & 87.45 & 102.02 & 115.38 & 127.53 & 140.89 & 151.42 & 161.54 & 176.11 & 166.80 \\
\hline 10.72 & 87.85 & 95.55 & 112.15 & 129.55 & 146.56 & 163.16 & 175.30 & 186.64 & 206.48 & 191.65 \\
\hline 12.33 & 94.33 & 104.05 & 121.86 & 144.94 & 168.02 & 189.07 & 203.64 & 215.79 & 240.08 & 222.92 \\
\hline 14.17 & 100.00 & 111.74 & 131.58 & 160.32 & 191.09 & 217.41 & 234.82 & 248.99 & 277.73 & 261.38 \\
\hline 16.30 & 103.64 & 118.22 & 140.49 & 174.49 & 213.36 & 247.77 & 269.64 & 287.04 & 324.29 & 307.70 \\
\hline
\end{tabular}




\begin{tabular}{|c|c|c|c|c|c|c|c|c|c|c|}
\hline 18.74 & 104.86 & 123.08 & 147.77 & 186.64 & 231.58 & 277.73 & 307.69 & 330.36 & 377.73 & 362.81 \\
\hline 21.54 & 102.43 & 124.29 & 151.82 & 195.14 & 246.15 & 304.86 & 345.75 & 377.73 & 437.25 & 426.47 \\
\hline 24.77 & 96.36 & 121.86 & 152.23 & 198.38 & 255.47 & 327.13 & 380.97 & 425.10 & 499.60 & 497.28 \\
\hline 28.48 & 87.45 & 116.19 & 148.58 & 197.17 & 258.30 & 343.72 & 412.15 & 470.04 & 562.35 & 572.38 \\
\hline 32.75 & 76.92 & 107.69 & 141.30 & 190.69 & 254.25 & 352.63 & 435.63 & 508.91 & 616.60 & 647.98 \\
\hline 37.65 & 65.59 & 96.76 & 131.17 & 179.76 & 244.53 & 353.85 & 449.39 & 538.87 & 662.75 & 719.18 \\
\hline 43.29 & 54.66 & 85.02 & 118.62 & 165.59 & 227.94 & 349.39 & 455.47 & 559.51 & 695.95 & 782.28 \\
\hline 49.77 & 44.94 & 73.28 & 104.86 & 148.99 & 206.88 & 335.63 & 452.63 & 570.45 & 717.00 & 834.75 \\
\hline 57.22 & 35.63 & 61.94 & 90.69 & 130.77 & 183.00 & 311.34 & 440.08 & 571.66 & 726.32 & 875.13 \\
\hline 65.79 & 27.13 & 50.61 & 76.11 & 112.96 & 157.89 & 280.57 & 417.41 & 562.35 & 724.70 & 902.11 \\
\hline 75.65 & 19.84 & 40.08 & 60.32 & 94.33 & 133.20 & 242.92 & 383.40 & 542.92 & 709.72 & 914.91 \\
\hline 86.97 & 13.36 & 30.36 & 45.75 & 76.52 & 109.72 & 200.41 & 336.44 & 511.74 & 679.76 & 912.32 \\
\hline 100.00 & 8.10 & 21.05 & 31.98 & 59.11 & 87.45 & 158.70 & 281.38 & 469.23 & 637.65 & 892.82 \\
\hline 114.98 & 4.45 & 12.96 & 19.84 & 42.11 & 66.80 & 121.86 & 223.48 & 415.79 & 578.14 & 855.18 \\
\hline 132.19 & 2.02 & 7.29 & 10.53 & 26.32 & 46.96 & 87.04 & 166.80 & 352.23 & 504.05 & 798.79 \\
\hline 151.99 & 0.40 & 3.24 & 4.86 & 14.98 & 29.96 & 58.70 & 116.19 & 283.40 & 417.81 & 724.14 \\
\hline 174.75 & 0.00 & 0.81 & 1.21 & 6.48 & 17.41 & 36.44 & 76.11 & 214.57 & 331.58 & 633.10 \\
\hline 200.92 & 0.00 & 0.00 & 0.00 & 1.62 & 8.50 & 20.24 & 46.56 & 150.61 & 245.34 & 530.10 \\
\hline 231.01 & 0.00 & 0.00 & 0.00 & 0.00 & 2.83 & 8.91 & 25.10 & 97.17 & 172.47 & 421.55 \\
\hline 265.61 & 0.00 & 0.00 & 0.00 & 0.00 & 0.81 & 3.24 & 11.74 & 57.49 & 111.74 & 315.39 \\
\hline 305.39 & 0.00 & 0.00 & 0.00 & 0.00 & 0.00 & 0.81 & 4.05 & 29.55 & 68.02 & 219.44 \\
\hline 351.12 & 0.00 & 0.00 & 0.00 & 0.00 & 0.00 & 0.00 & 1.21 & 13.36 & 36.44 & 140.25 \\
\hline 403.70 & 0.00 & 0.00 & 0.00 & 0.00 & 0.00 & 0.00 & 0.00 & 5.26 & 18.22 & 81.00 \\
\hline 464.16 & 0.00 & 0.00 & 0.00 & 0.00 & 0.00 & 0.00 & 0.00 & 1.21 & 6.48 & 41.31 \\
\hline 533.67 & 0.00 & 0.00 & 0.00 & 0.00 & 0.00 & 0.00 & 0.00 & 0.00 & 2.02 & 17.94 \\
\hline 613.59 & 0.00 & 0.00 & 0.00 & 0.00 & 0.00 & 0.00 & 0.00 & 0.00 & 0.00 & 6.26 \\
\hline 705.48 & 0.00 & 0.00 & 0.00 & 0.00 & 0.00 & 0.00 & 0.00 & 0.00 & 0.00 & 1.55 \\
\hline 811.13 & 0.00 & 0.00 & 0.00 & 0.00 & 0.00 & 0.00 & 0.00 & 0.00 & 0.00 & 0.19 \\
\hline
\end{tabular}




\begin{tabular}{|l|l|l|l|l|l|l|l|l|l|l|}
\hline 932.60 & 0.00 & 0.00 & 0.00 & 0.00 & 0.00 & 0.00 & 0.00 & 0.00 & 0.00 & 0.00 \\
\hline 1072.27 & 0.00 & 0.00 & 0.00 & 0.00 & 0.00 & 0.00 & 0.00 & 0.00 & 0.00 & 0.00 \\
\hline 1232.85 & 0.00 & 0.00 & 0.00 & 0.00 & 0.00 & 0.00 & 0.00 & 0.00 & 0.00 & 0.00 \\
\hline 1417.47 & 0.00 & 0.00 & 0.00 & 0.00 & 0.00 & 0.00 & 0.00 & 0.00 & 0.00 & 0.00 \\
\hline 1629.75 & 0.00 & 0.00 & 0.00 & 0.00 & 0.00 & 0.00 & 0.00 & 0.00 & 0.00 & 0.00 \\
\hline 1873.82 & 0.00 & 0.00 & 0.00 & 0.00 & 0.00 & 0.00 & 0.00 & 0.00 & 0.00 & 0.00 \\
\hline 2154.43 & 0.00 & 0.00 & 0.00 & 0.00 & 0.00 & 0.00 & 0.00 & 0.00 & 0.00 & 0.00 \\
\hline 2477.08 & 0.00 & 0.00 & 0.00 & 0.00 & 0.00 & 0.00 & 0.00 & 0.00 & 0.00 & 0.00 \\
\hline 2848.04 & 0.00 & 0.00 & 0.00 & 0.00 & 0.00 & 0.00 & 0.00 & 0.00 & 0.00 & 0.00 \\
\hline 3274.55 & 0.00 & 0.00 & 0.00 & 0.00 & 0.00 & 0.00 & 0.00 & 0.00 & 0.00 & 0.00 \\
\hline 3764.94 & 0.00 & 0.00 & 0.00 & 0.00 & 0.00 & 0.00 & 0.00 & 0.00 & 0.00 & 0.00 \\
\hline 4328.76 & 0.00 & 0.00 & 0.00 & 0.00 & 0.00 & 0.00 & 0.00 & 0.00 & 0.00 & 0.00 \\
\hline 4977.02 & 0.00 & 0.00 & 0.00 & 0.00 & 0.00 & 0.00 & 0.00 & 0.00 & 0.00 & 0.00 \\
\hline 5722.37 & 0.00 & 0.00 & 0.00 & 0.00 & 0.00 & 0.00 & 0.00 & 0.00 & 0.00 & 0.00 \\
\hline 6579.33 & 0.00 & 0.00 & 0.00 & 0.00 & 0.00 & 0.00 & 0.00 & 0.00 & 0.00 & 0.00 \\
\hline 7564.63 & 0.00 & 0.00 & 0.00 & 0.00 & 0.00 & 0.00 & 0.00 & 0.00 & 0.00 & 0.00 \\
\hline 8697.49 & 0.00 & 0.00 & 0.00 & 0.00 & 0.00 & 0.00 & 0.00 & 0.00 & 0.00 & 0.00 \\
\hline 10000.00 & 0.00 & 0.00 & 0.00 & 0.00 & 0.00 & 0.00 & 0.00 & 0.00 & 0.00 & 0.00 \\
\hline
\end{tabular}


Table S4: Data of T2 spectra obtained from different hydrate dissociation time for Sample 3

\begin{tabular}{|c|c|c|c|c|c|c|c|c|c|c|}
\hline Time(ms) & D1 & D2 & D3 & D4 & D5 & D6 & D7 & D8 & D9 & D10 \\
\hline 0.01 & 0.00 & 0.00 & 0.00 & 0.00 & 0.00 & 0.00 & 0.00 & 0.00 & 0.00 & 0.00 \\
\hline 0.01 & 0.00 & 0.00 & 0.00 & 0.00 & 0.00 & 0.00 & 0.00 & 0.00 & 0.00 & 0.00 \\
\hline 0.01 & 0.00 & 0.00 & 0.00 & 0.00 & 0.00 & 0.00 & 0.00 & 0.00 & 0.00 & 0.00 \\
\hline 0.02 & 0.00 & 0.00 & 0.00 & 0.00 & 0.00 & 0.00 & 0.00 & 0.00 & 0.00 & 0.00 \\
\hline 0.02 & 0.00 & 0.00 & 0.00 & 0.00 & 0.00 & 0.00 & 0.00 & 0.00 & 0.00 & 0.00 \\
\hline 0.02 & 0.00 & 0.00 & 0.00 & 0.00 & 0.00 & 0.00 & 0.00 & 0.00 & 0.00 & 0.00 \\
\hline 0.02 & 0.00 & 0.00 & 0.00 & 0.00 & 0.00 & 0.00 & 0.00 & 0.00 & 0.00 & 0.00 \\
\hline 0.03 & 0.00 & 0.00 & 0.00 & 0.00 & 0.00 & 0.00 & 0.00 & 0.00 & 0.00 & 0.00 \\
\hline 0.03 & 0.00 & 0.00 & 0.00 & 0.00 & 0.00 & 0.00 & 0.00 & 0.00 & 0.00 & 0.00 \\
\hline 0.04 & 0.00 & 0.00 & 0.00 & 0.00 & 0.00 & 0.00 & 0.00 & 0.00 & 0.00 & 0.00 \\
\hline 0.04 & 0.00 & 0.00 & 0.00 & 0.00 & 0.00 & 0.00 & 0.00 & 0.00 & 0.00 & 0.00 \\
\hline 0.05 & 0.00 & 0.00 & 0.00 & 0.00 & 0.00 & 0.00 & 0.00 & 0.00 & 0.00 & 0.00 \\
\hline 0.05 & 0.00 & 0.00 & 0.00 & 0.00 & 0.00 & 0.00 & 0.00 & 0.00 & 0.00 & 0.00 \\
\hline 0.06 & 0.00 & 0.00 & 0.00 & 0.00 & 0.00 & 0.00 & 0.00 & 0.00 & 0.00 & 0.00 \\
\hline 0.07 & 0.00 & 0.00 & 0.00 & 0.00 & 0.00 & 0.00 & 0.00 & 0.00 & 0.00 & 0.00 \\
\hline 0.08 & 0.00 & 0.00 & 0.00 & 0.00 & 0.00 & 0.00 & 0.00 & 0.00 & 0.00 & 0.00 \\
\hline 0.09 & 0.00 & 0.00 & 0.00 & 0.00 & 0.00 & 0.00 & 0.00 & 0.00 & 0.00 & 0.00 \\
\hline 0.11 & 0.00 & 0.00 & 0.00 & 0.00 & 0.00 & 0.00 & 0.00 & 0.00 & 0.00 & 0.00 \\
\hline 0.12 & 0.00 & 0.00 & 0.00 & 0.00 & 0.00 & 0.00 & 0.00 & 0.00 & 0.00 & 0.00 \\
\hline 0.14 & 0.00 & 0.00 & 0.00 & 0.00 & 0.00 & 0.00 & 0.00 & 0.00 & 0.00 & 0.00 \\
\hline 0.16 & 0.00 & 0.00 & 0.00 & 0.00 & 0.00 & 0.00 & 0.00 & 0.00 & 0.65 & 2.46 \\
\hline 0.19 & 0.00 & 0.00 & 0.00 & 0.00 & 0.00 & 0.00 & 0.00 & 0.65 & 2.59 & 6.77 \\
\hline 0.22 & 0.00 & 0.00 & 0.00 & 0.00 & 0.00 & 0.00 & 1.30 & 2.59 & 5.84 & 12.31 \\
\hline 0.25 & 0.00 & 0.00 & 0.00 & 0.00 & 0.00 & 1.30 & 3.89 & 5.84 & 10.38 & 19.08 \\
\hline 0.28 & 0.00 & 0.00 & 0.00 & 0.00 & 1.95 & 3.89 & 7.78 & 10.38 & 15.57 & 26.46 \\
\hline 0.33 & 0.00 & 0.00 & 0.65 & 1.95 & 5.19 & 7.78 & 12.97 & 16.22 & 21.41 & 32.62 \\
\hline
\end{tabular}




\begin{tabular}{|c|c|c|c|c|c|c|c|c|c|c|}
\hline 0.38 & 0.00 & 1.30 & 2.59 & 5.19 & 9.73 & 12.97 & 19.46 & 22.70 & 27.24 & 38.15 \\
\hline 0.43 & 1.30 & 3.24 & 5.19 & 9.08 & 14.92 & 18.81 & 25.30 & 28.54 & 32.43 & 43.69 \\
\hline 0.50 & 3.89 & 6.49 & 9.08 & 14.27 & 21.41 & 24.65 & 31.14 & 34.38 & 38.27 & 49.85 \\
\hline 0.57 & 7.78 & 11.03 & 14.27 & 20.76 & 27.24 & 30.49 & 36.97 & 40.22 & 44.11 & 56.62 \\
\hline 0.66 & 13.62 & 17.51 & 20.76 & 27.24 & 33.73 & 36.97 & 43.46 & 46.70 & 50.59 & 64.00 \\
\hline 0.76 & 22.05 & 25.30 & 28.54 & 35.03 & 41.51 & 44.76 & 51.24 & 54.49 & 58.38 & 72.00 \\
\hline 0.87 & 32.43 & 35.68 & 38.92 & 45.41 & 51.89 & 55.14 & 60.97 & 64.22 & 68.11 & 81.85 \\
\hline 1.00 & 44.76 & 48.00 & 51.24 & 57.73 & 64.22 & 67.46 & 72.65 & 75.89 & 79.14 & 92.92 \\
\hline 1.15 & 58.38 & 61.62 & 64.86 & 71.35 & 77.19 & 80.43 & 84.97 & 88.22 & 91.46 & 105.23 \\
\hline 1.32 & 72.65 & 75.89 & 79.14 & 84.97 & 90.16 & 93.41 & 97.30 & 100.54 & 103.78 & 117.54 \\
\hline 1.52 & 85.62 & 88.86 & 92.11 & 97.30 & 101.84 & 105.08 & 108.32 & 111.57 & 114.81 & 129.23 \\
\hline 1.75 & 95.35 & 98.59 & 101.84 & 106.38 & 110.27 & 113.51 & 116.76 & 120.00 & 123.24 & 138.46 \\
\hline 2.01 & 101.19 & 104.43 & 107.68 & 111.57 & 115.46 & 119.35 & 122.59 & 125.84 & 129.08 & 145.23 \\
\hline 2.31 & 103.14 & 107.03 & 110.27 & 114.16 & 118.70 & 123.24 & 126.49 & 129.73 & 132.97 & 149.54 \\
\hline 2.66 & 99.24 & 103.78 & 107.68 & 113.51 & 119.35 & 125.19 & 128.43 & 131.68 & 134.92 & 152.00 \\
\hline 3.05 & 92.11 & 97.95 & 102.49 & 110.27 & 117.41 & 125.19 & 129.73 & 132.97 & 136.22 & 153.23 \\
\hline 3.51 & 83.03 & 90.16 & 95.35 & 105.73 & 114.16 & 124.54 & 131.03 & 134.27 & 137.51 & 154.46 \\
\hline 4.04 & 72.00 & 80.43 & 86.27 & 99.89 & 109.62 & 121.95 & 131.03 & 135.57 & 140.11 & 156.92 \\
\hline 4.64 & 60.97 & 70.05 & 77.19 & 92.76 & 104.43 & 118.70 & 131.03 & 137.51 & 143.35 & 160.62 \\
\hline 5.34 & 52.54 & 62.27 & 70.05 & 85.62 & 99.24 & 116.11 & 131.68 & 140.76 & 149.84 & 166.15 \\
\hline 6.14 & 46.05 & 55.78 & 64.22 & 79.78 & 96.00 & 115.46 & 133.62 & 145.95 & 157.62 & 173.54 \\
\hline 7.05 & 44.11 & 53.84 & 62.92 & 78.49 & 96.65 & 118.05 & 137.51 & 153.08 & 166.70 & 182.15 \\
\hline 8.11 & 47.35 & 57.08 & 67.46 & 82.38 & 101.84 & 124.54 & 144.65 & 161.51 & 176.43 & 191.38 \\
\hline 9.33 & 54.49 & 65.51 & 76.54 & 91.46 & 110.92 & 134.27 & 154.38 & 172.54 & 189.41 & 202.46 \\
\hline 10.72 & 66.81 & 79.14 & 90.81 & 107.03 & 125.84 & 147.89 & 168.00 & 185.51 & 201.73 & 215.38 \\
\hline 12.33 & 84.97 & 97.95 & 110.92 & 129.08 & 145.95 & 165.41 & 184.22 & 200.43 & 216.00 & 230.15 \\
\hline 14.17 & 106.38 & 120.65 & 134.92 & 153.73 & 169.30 & 186.16 & 204.32 & 219.24 & 234.16 & 248.60 \\
\hline 16.30 & 131.68 & 147.24 & 162.16 & 180.97 & 195.89 & 210.81 & 227.68 & 241.95 & 256.22 & 272.75 \\
\hline
\end{tabular}




\begin{tabular}{|c|c|c|c|c|c|c|c|c|c|c|}
\hline 18.74 & 158.27 & 173.84 & 189.41 & 208.87 & 224.43 & 240.00 & 255.57 & 268.54 & 282.81 & 303.05 \\
\hline 21.54 & 186.81 & 201.73 & 217.95 & 237.41 & 254.27 & 272.43 & 287.35 & 302.27 & 319.10 & 346.12 \\
\hline 24.77 & 217.30 & 232.22 & 250.38 & 269.84 & 289.30 & 312.00 & 332.76 & 352.22 & 376.45 & 410.19 \\
\hline 28.48 & 249.08 & 263.35 & 283.46 & 304.87 & 330.81 & 360.00 & 391.78 & 419.76 & 452.84 & 493.02 \\
\hline 32.75 & 282.16 & 297.73 & 321.08 & 345.73 & 379.46 & 420.97 & 467.03 & 506.06 & 548.31 & 592.23 \\
\hline 37.65 & 319.78 & 337.30 & 363.24 & 393.08 & 435.89 & 493.62 & 557.84 & 609.92 & 661.34 & 708.29 \\
\hline 43.29 & 360.00 & 378.16 & 410.59 & 448.86 & 504.00 & 577.95 & 660.97 & 727.13 & 787.46 & 834.51 \\
\hline 49.77 & 398.92 & 419.68 & 459.24 & 511.14 & 575.35 & 667.46 & 764.11 & 845.16 & 912.33 & 962.03 \\
\hline 57.22 & 433.95 & 460.54 & 510.49 & 576.00 & 647.35 & 756.97 & 864.65 & 955.63 & 1033.86 & 1090.48 \\
\hline 65.79 & 457.95 & 493.62 & 556.54 & 637.62 & 716.11 & 838.05 & 954.16 & 1055.61 & 1146.82 & 1215.95 \\
\hline 75.65 & 467.68 & 513.73 & 591.57 & 688.86 & 776.43 & 907.46 & 1029.41 & 1140.01 & 1245.96 & 1330.62 \\
\hline 86.97 & 459.89 & 520.86 & 610.38 & 720.65 & 813.41 & 954.16 & 1081.95 & 1204.15 & 1326.26 & 1426.68 \\
\hline 100.00 & 434.59 & 513.73 & 611.68 & 726.49 & 828.32 & 974.92 & 1110.49 & 1244.07 & 1383.24 & 1499.42 \\
\hline 114.98 & 391.14 & 491.68 & 592.86 & 710.27 & 819.24 & 971.03 & 1111.79 & 1259.26 & 1413.29 & 1544.42 \\
\hline 132.19 & 338.59 & 453.41 & 557.19 & 673.30 & 789.41 & 940.54 & 1087.79 & 1245.52 & 1413.96 & 1560.42 \\
\hline 151.99 & 282.16 & 402.16 & 509.19 & 618.81 & 739.46 & 884.11 & 1036.54 & 1202.38 & 1384.18 & 1546.08 \\
\hline 174.75 & 221.19 & 338.59 & 449.51 & 551.35 & 676.54 & 810.81 & 964.54 & 1130.93 & 1324.41 & 1504.33 \\
\hline 200.92 & 159.57 & 264.65 & 376.22 & 474.16 & 599.35 & 723.89 & 871.31 & 1034.25 & 1236.65 & 1433.66 \\
\hline 231.01 & 107.03 & 190.05 & 295.78 & 388.54 & 509.84 & 621.41 & 760.12 & 916.26 & 1124.36 & 1334.92 \\
\hline 265.61 & 64.22 & 122.59 & 212.11 & 298.38 & 406.70 & 509.19 & 634.87 & 782.41 & 992.19 & 1209.95 \\
\hline 305.39 & 31.14 & 68.76 & 136.22 & 211.46 & 301.62 & 393.08 & 502.20 & 639.18 & 845.62 & 1065.74 \\
\hline 351.12 & 11.03 & 33.08 & 75.24 & 133.62 & 199.78 & 280.22 & 372.61 & 496.12 & 690.49 & 907.13 \\
\hline 403.70 & 2.59 & 13.62 & 34.38 & 73.95 & 116.76 & 178.38 & 255.24 & 360.04 & 532.54 & 742.43 \\
\hline 464.16 & 0.00 & 3.24 & 11.03 & 33.73 & 57.08 & 99.89 & 154.97 & 231.62 & 376.90 & 578.11 \\
\hline 533.67 & 0.00 & 0.00 & 1.95 & 10.38 & 21.41 & 46.70 & 83.03 & 133.92 & 242.22 & 419.57 \\
\hline 613.59 & 0.00 & 0.00 & 0.00 & 0.65 & 3.24 & 16.22 & 37.32 & 65.86 & 136.35 & 274.24 \\
\hline 705.48 & 0.00 & 0.00 & 0.00 & 0.00 & 0.00 & 2.59 & 11.24 & 22.49 & 62.49 & 160.02 \\
\hline 811.13 & 0.00 & 0.00 & 0.00 & 0.00 & 0.00 & 0.00 & 0.00 & 3.26 & 19.05 & 78.12 \\
\hline
\end{tabular}




\begin{tabular}{|l|l|l|l|l|l|l|l|l|l|l|}
\hline 932.60 & 0.00 & 0.00 & 0.00 & 0.00 & 0.00 & 0.00 & 0.00 & 0.00 & 3.88 & 26.47 \\
\hline 1072.27 & 0.00 & 0.00 & 0.00 & 0.00 & 0.00 & 0.00 & 0.00 & 0.00 & 0.00 & 2.69 \\
\hline 1232.85 & 0.00 & 0.00 & 0.00 & 0.00 & 0.00 & 0.00 & 0.00 & 0.00 & 0.00 & 0.00 \\
\hline 1417.47 & 0.00 & 0.00 & 0.00 & 0.00 & 0.00 & 0.00 & 0.00 & 0.00 & 0.00 & 0.00 \\
\hline 1629.75 & 0.00 & 0.00 & 0.00 & 0.00 & 0.00 & 0.00 & 0.00 & 0.00 & 0.00 & 0.00 \\
\hline 1873.82 & 0.00 & 0.00 & 0.00 & 0.00 & 0.00 & 0.00 & 0.00 & 0.00 & 0.00 & 0.00 \\
\hline 2154.43 & 0.00 & 0.00 & 0.00 & 0.00 & 0.00 & 0.00 & 0.00 & 0.00 & 0.00 & 0.00 \\
\hline 2477.08 & 0.00 & 0.00 & 0.00 & 0.00 & 0.00 & 0.00 & 0.00 & 0.00 & 0.00 & 0.00 \\
\hline 2848.04 & 0.00 & 0.00 & 0.00 & 0.00 & 0.00 & 0.00 & 0.00 & 0.00 & 0.00 & 0.00 \\
\hline 3274.55 & 0.00 & 0.00 & 0.00 & 0.00 & 0.00 & 0.00 & 0.00 & 0.00 & 0.00 & 0.00 \\
\hline 3764.94 & 0.00 & 0.00 & 0.00 & 0.00 & 0.00 & 0.00 & 0.00 & 0.00 & 0.00 & 0.00 \\
\hline 4328.76 & 0.00 & 0.00 & 0.00 & 0.00 & 0.00 & 0.00 & 0.00 & 0.00 & 0.00 & 0.00 \\
\hline 4977.02 & 0.00 & 0.00 & 0.00 & 0.00 & 0.00 & 0.00 & 0.00 & 0.00 & 0.00 & 0.00 \\
\hline 5722.37 & 0.00 & 0.00 & 0.00 & 0.00 & 0.00 & 0.00 & 0.00 & 0.00 & 0.00 & 0.00 \\
\hline 6579.33 & 0.00 & 0.00 & 0.00 & 0.00 & 0.00 & 0.00 & 0.00 & 0.00 & 0.00 & 0.00 \\
\hline 7564.63 & 0.00 & 0.00 & 0.00 & 0.00 & 0.00 & 0.00 & 0.00 & 0.00 & 0.00 & 0.00 \\
\hline 8697.49 & 0.00 & 0.00 & 0.00 & 0.00 & 0.00 & 0.00 & 0.00 & 0.00 & 0.00 & 0.00 \\
\hline 10000.00 & 0.00 & 0.00 & 0.00 & 0.00 & 0.00 & 0.00 & 0.00 & 0.00 & 0.00 & 0.00 \\
\hline
\end{tabular}


Table S5: Calculation results of the fractal dimension for the three samples

\begin{tabular}{|c|c|c|c|}
\hline Dissociation stage & Sample 1 & Sample 2 & 2.44 \\
\hline D1 & 2.68 & 2.59 & 2.40 \\
\hline D2 & 2.45 & 2.57 & 2.35 \\
\hline D3 & 2.36 & 2.53 & 2.27 \\
\hline D4 & 2.31 & 2.50 & 2.21 \\
\hline D5 & 2.29 & 2.46 & 2.17 \\
\hline D6 & 2.25 & 2.43 & 2.15 \\
\hline D7 & 2.19 & 2.37 & 2.10 \\
\hline D8 & 2.14 & 2.32 & 2.06 \\
\hline D9 & 2.10 & 2.27 & 2.09 \\
\hline
\end{tabular}

